\title{
BMJ Open Prevalence of papillomavirus in Brazil: a systematic review protocol
}

\author{
Verônica Colpani, ${ }^{1}$ Augusto Bacelo Bidinotto, ${ }^{1,2}$ Maicon Falavigna, ${ }^{3}$ \\ Silvana Pereira Giozza, ${ }^{4}$ Adele Schwartz Benzaken, ${ }^{4}$ Cristina Pimenta, ${ }^{4}$ \\ Ana Goretti Kalume Maranhão, ${ }^{5}$ Carla Magda Allan Santos Domingues, ${ }^{5}$ \\ Luciano Serpa Hammes, ${ }^{6}$ Eliana M Wendland ${ }^{6}$
}

To cite: Colpani V, Bidinotto $A B$, Falavigna $M$, et al. Prevalence of papillomavirus in Brazil: a systematic review protocol. BMJ Open 2016;6:e011884. doi:10.1136/bmjopen-2016011884

- Prepublication history and additional material is available. To view please visit the journal (http://dx.doi.org/ 10.1136/bmjopen-2016011884).

Received 11 March 2016 Revised 17 September 2016 Accepted 30 September 2016

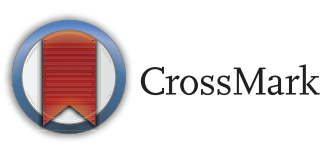

For numbered affiliations see end of article.

Correspondence to Dr Eliana M Wendland; eliana.wendland@hmv.org.br

\section{ABSTRACT}

Introduction: Human papillomavirus (HPV) infection is a cause of premalignant and malignant cancer in the lower genital and digestive tracts. In Brazil, there have been no prevalence studies that included a nationwide sample, and the prevalence of HPV has not been determined in many regions.

Methods: We will search the EMBASE, LILACS, MEDLINE, Web of Science and SciELO databases and previously published review articles to identify original research articles assessing HPV prevalence of the perineal (cervical, penile and anal) and oral areas. No exclusion criteria related to language or publication date will apply. 2 reviewers will independently screen for eligibility and perform data extraction.

Discrepancies will be resolved through consensus; the opinion of a third reviewer will be sought as necessary. Relevant measures and data about study and population characteristics will be extracted from the included studies. Where possible, study prevalence will be pooled using a random-effects meta-analysis. The methodological quality of the studies will be assessed using an adapted version of the NIH 'Quality Assessment Tool for Observational Cohort and CrossSectional Studies'. The overall quality of evidence will be assessed using Grading of Recommendations Assessment, Development and Evaluation (GRADE).

Ethics and dissemination: We expect to estimate the prevalence of perineal and oral HPV infection in the general population as well as the prevalence of HPV infection in individuals with premalignant and malignant lesions in Brazil and its 5 geographic regions. This systematic review does not require ethical approval.

Trial registration number: CRD42016032751.

\section{INTRODUCTION}

Cervical cancer is the fourth most frequent type of cancer among Brazilian women, excluding non-melanoma skin tumours. The crude incidence rate for 2014 has been estimated at 15.3 cases $/ 100000$ women. However, the prevalence varies in different

\section{Strengths and limitations of this study}

- To the best of our knowledge, this is the first systematic review evaluating the overall human papillomavirus prevalence in Brazil, stratified by geographic region.

- This study could potentially inform the gaps in the literature and assess the need for a new original study with a nationwide sample.

- The quality of evidence for all outcomes will be judged using the Grading of Recommendations Assessment, Development and Evaluation (GRADE) approach.

- We anticipate that there may be variations across studies related to sample size, participants and sociodemographic regional differences. We will control for this heterogeneity with the statistical analyses described in the methods section.

Brazilian geographic regions and is higher in the North and Central-West. ${ }^{1}$

Oral cancer is the fourth most common type of cancer among men in the Southeast and Northeast Brazilian regions (excluding non-melanoma skin tumours). In Brazil, in 2014, the incidence of oral cancer in men was 11.54 cases $/ 100000$, and in women it was $3.92 / 100000{ }^{1}$

The human papillomavirus (HPV) has been found to be a cause of premalignant and malignant tumours in the lower genital and digestive tracts. ${ }^{2}$ There are more than $100 \mathrm{HPV}$ types, ${ }^{3}$ and the most carcinogenic types affecting the cervix are the following: $16,18,31,33,35,39,45,51,52,56,58$, and $59 .{ }^{4}$ HPV infection accounts for more than $99 \%$ of cases of cervical cancer, whereas nononcogenic HPV types, such as 6 and 11, are responsible for $90 \%$ of anogenital warts (papilloma) ${ }^{5-7} \mathrm{HPV}$ is also associated with oropharyngeal cancer. ${ }^{8} \quad 9$ Approximately $90 \%$ of HPV-positive oropharyngeal squamous cell carcinomas are associated with HPV type $16 .{ }^{10}$

The global prevalence of HPV infection is $\sim 12 \%$, with substantial regional variation. ${ }^{11} 12$ 
In the USA, the prevalence of HPV is $42.5 \%$ in women aged 14-59 years. ${ }^{7}$ A previous review on cervical HPV infection in Brazil showed an overall prevalence of cervical HPV infection ranging from $13.7 \%$ to $54.3 \%$ in the general population and from $10 \%$ to $24.5 \%$ among women with normal cytological results. ${ }^{5}$ Although there is limited information available about HPV prevalence in other parts of the body, such as oral and oesophageal, a recent review of 4581 cancer-free participants showed a $4.5 \%(95 \%$ CI $3.9 \%$ to $5.1 \%$ ) prevalence of oral HPV infection in the USA. ${ }^{13}$

The absence of a national database and screening for precancerous lesions imposes limitations on the planning of surveillance strategies and hinders primary healthcare prevention. Additionally, HPV vaccination in Brazil began in 2014, using the extended schedule $(0,6$ and 60 months), in girls aged 9-13 years. Understanding the prevalence of HPV prior to vaccination will be useful for monitoring the national vaccination programme's impact and evaluating changes in the differential regional distribution of HPV in Brazil.

Therefore, the primary objective of this systematic review will be to assess the prevalence of genito-anal area (cervical, penile and anal region) and oral HPV infection in the general population in Brazil. The secondary objective is to evaluate the prevalence of HPV infection in individuals with premalignant and malignant lesions in Brazil.

\section{METHODS AND ANALYSIS}

\section{Protocol and registration}

This systematic review protocol is prepared according to the Preferred Reporting Items for Systematic reviews and Meta-Analysis Protocols (PRISMA-P) statement (see online supplementary additional file 1$).{ }^{14}$ This review protocol has been registered in the PROSPERO International Prospective Register of systematic reviews (http://www.crd.york.ac.uk/PROSPERO), registration number CRD42016032751.

\section{Literature search}

We will search the following databases: EMBASE, LILACS, MEDLINE, Web of Science and SciELO. Search terms will include relevant headings and keywords in the title, abstract and text, including human papillomavirus, prevalence and Brazil. The search strategy is presented in online supplementary additional file 2. This search strategy will be performed in cooperation with a research librarian.

We will evaluate the reference lists of identified publications for additional studies. A Brazilian specialist in the area will be contacted to identify unpublished and ongoing studies. Additionally, we will use the website 'bancodeteses.capes.gov.br' to identify dissertations in the area, and websites such as the Grey Literature Report (http://www.greylit.org) will be searched as grey literature. Additionally, the Department of IST/HIV has an extensive scientific network, and personal information from experts in the area will be personally gathered. Data from conference proceedings will be included, even without an answer from the authors, if the abstract provides enough information to assess its eligibility and to collect at least the overall prevalence and number of participants.

\section{Eligible criteria}

This systematic review will include studies meeting the following criteria: (1) randomised controlled trials, cohort studies, cross-sectional studies and prevalence studies; (2) studies evaluating the prevalence of perineal (cervical, penile and anal region) and oral HPV infection in Brazil; (3) HPV assessments through PCR, hybrid capture or any described genotyping methodology. We will exclude studies evaluating only pregnant women and studies that analysed material such as blood, sperm and urine. No gender, age, language or date restrictions will be applied.

\section{Study selection and data extraction}

Two independent reviewers will screen the titles and abstracts of all studies initially identified, according to the selection criteria. The same researchers will independently perform data extraction for full text review, using standardised forms. Discrepancies will be resolved through consensus; the opinion of a third reviewer will be sought if necessary. The following data will be collected: publication title, authors, publication year, study design, characteristics of the population (number of participants, age, gender, race, educational level, sexual history information, comorbidities and geographical region in Brazil), number of HPV-positive and HPV-negative women, HPV type, HPV detection and genotyping methodology, HPV vaccination history, HPV risk factors (ie, oral sex, multiple sexual partners) and the presence of HIV. For cohort and randomised studies, only the baseline characteristics of the population will be extracted. For systematic reviews, we will only scan the reference lists for additional studies. If the study is reported in duplicate, the study published earlier or the one that provides more information will be included. An email will be sent to the authors of eligible duplicated studies to ask for additional details about these articles.

\section{Risk of bias (quality) assessment}

Once all searches have been completed, the included studies will be assessed for quality using an adapted version of the NIH 'Quality Assessment Tool for Observational Cohort and Cross-Sectional Studies'. ${ }^{15}$ The overall quality of evidence will be assessed using Grading of Recommendations Assessment, Development and Evaluation (GRADE), using the same principles and domains applied in the quality assessment for prognostic studies. ${ }^{16}$ 


\section{Strategy for data synthesis}

We will provide a clear narrative synthesis and summary tables of the findings from the included studies, structured around the HPV prevalence. When possible, data will be pooled using a meta-analytic approach. The random-effects model will be used to calculate pooled prevalence and corresponding 95\% CI. If possible, the prevalence of HPV infection in Brazil will be adjusted, considering the population of each one of the five main Brazilian regions (North, Northeast, Centre-West, Southeast and South). The heterogeneity of the findings will be assessed using both the $\chi^{2}$ test and the $\mathrm{I}^{2}$ statistic. Sensitivity analysis will be performed by subgroup analyses and meta-regression to investigate the effect of study-level characteristics, such as age, gender, HPV type and methodological quality score, whenever possible. Publication bias across studies will be evaluated using funnel plots and Begg's tests. We will perform subgroup analysis for geographic area (Brazilian region), body region (cervical, penile, anal and oral regions), method of HPV assessment, HIV co-infection and the presence of premalignant or malignant lesions. If possible, we will also perform subgroup analyses for age, gender and HPV type. Studies in which the HPV type (high or low risk) was not assessed or was not reported will be excluded from the HPV type-specific analyses. A table with the characteristics of studies included will be used to present the results, and the GRADE approach will be used to assess the quality of the body of evidence. Analysis will be performed using R, V.3.2.3, packages meta V.4.3-2 and metaphor. ${ }^{17} 18$

\section{ETHICS AND DISSEMINATION}

In Brazil, HPV vaccination started in 2014 and is provided by the National Health System Sistema Único de Saúde (SUS). It is expected that the vaccination programme will decrease HPV infection and HPV-related cancer, improving healthcare and reducing healthcare costs. Prevalence studies are often used to plan public health actions and evaluate national control programmes. There are no HPV prevalence studies with a nationwide sample in Brazil. Single studies show heterogeneous results, assessing populations from different geographic areas, with different baseline risks and using diverse methods for HPV assessment.

The previous review on cervical HPV prevalence ${ }^{5}$ published in Brazil in 2010 searched few databases, did not include the HIV-positive population and did not analyse premalignant and malignant lesions. Another review on oral HPV was published in 2015 but had language restrictions. ${ }^{19}$ No systematic review has been conducted to estimate the overall prevalence of HPV infection in Brazil.

With this systematic review, we expect to estimate the prevalence of HPV in Brazil, to identify gaps in the literature and to assess the need for a new original study with a nationwide sample. The results of this systematic review will be used to compare the prevalence of HPV in different geographical regions of the country as well as the characteristics of the studied populations.

However, it is unlikely that this systematic review will end with sufficient evidence on the national prevalence of HPV to provide adequate baseline data to monitor the effect of the Brazilian HPV vaccination programme over time. To meet the Brazilian Ministry of Health needs, we have already started a working group to design and conduct a nationwide study to determine the prevalence of HPV. The Prevalence of Papillomavirus in Brazil (POP-Brazil) study will investigate the prevalence of HPV infection in men and women aged 16-25 years, from primary healthcare units, in all state capital cities in Brazil. The present review will provide support for the development and organisation of the POP-Brazil study and show the gaps in the evaluation of HPV prevalence on a national magnitude.

\section{Author affiliations}

${ }^{1}$ Hospital Moinhos de Vento, Porto Alegre, Brazil

${ }^{2}$ Post-Graduate Program in Epidemiology, Federal University of Rio Grande do Sul, Porto Alegre, Brazil

${ }^{3}$ Hospital Moinhos de Vento, Porto Alegre, Brazil

${ }^{4}$ Department of STDs/AIDS and Viral Hepatitis, Health Surveillance Secretariat, Ministry of Health, Brasilia, Brazil

${ }^{5}$ National Program of Immunization/Health Surveillance Secretariat, Ministry of Health, Brasilia, Brazil

${ }^{6}$ Hospital Moinhos de Vento, Porto Alegre, Brazil

Acknowledgements The authors thank Jeanine Rocha, who provided administrative support and edited the scientific language for reviewing this manuscript.

Contributors VC and EMW wrote the protocol. VC and ABB will individually perform the abstract extraction and critique the literature, and EMW will be the third reviewer. MF provided insight on the epidemiological aspects of the review and helped draft the manuscript. VC, ABB, EMW, MF, SPG, ASB, CP, AGKM, CMASD and LSH advised on background and revised the manuscript. All authors approve the final version and take responsibility for its content. The guarantor of the review is EMW.

Funding This work was supported by the Brazilian Ministry of Health, National Program of Immunizations through the Pan American Health Organization, project number BR/CNT/1500013.

Competing interests None declared.

Provenance and peer review Not commissioned; externally peer reviewed.

Open Access This is an Open Access article distributed in accordance with the Creative Commons Attribution Non Commercial (CC BY-NC 4.0) license, which permits others to distribute, remix, adapt, build upon this work noncommercially, and license their derivative works on different terms, provided the original work is properly cited and the use is non-commercial. See: http:// creativecommons.org/licenses/by-nc/4.0/

\section{REFERENCES}

1. INCA. Estimativa 2014: Incidência de Câncer no Brasil. 2014. http://www.saude.sp.gov.br/resources/ses/perfil/gestor/homepage/ outros-destaques/estimativa-de-incidencia-de-cancer-2014/ estimativa_cancer_24042014.pdf (accessed 28 Dec 2015).

2. Goodman A. HPV testing as a screen for cervical cancer. BMJ 2015;350:h2372.

3. Coser J, da Rocha Boeira T, Simon D, et al. Prevalence and genotypic diversity of cervical human papillomavirus infection among women from an urban center in Brazil. Genet Mol Res 2013;12:4276-85. 
4. Bouvard V, Baan $\mathrm{R}$, Straif $\mathrm{K}$, et al. A review of human carcinogens-part B: biological agents. Lancet Oncol 2009;10:321-2.

5. Ayres AR, Silva GA. Cervical HPV infection in Brazil: systematic review. Rev Saude Publica 2010;44:963-74.

6. Bosch FX, Tsu V, Vorsters A, et al. Reframing cervical cancer prevention. Expanding the field towards prevention of human papillomavirus infections and related diseases. Vaccine 2012;30: F1-11.

7. Hariri S, Unger ER, Sternberg M, et al. Prevalence of genital human papillomavirus among females in the United States, the National Health And Nutrition Examination Survey, 2003-2006. J Infect Dis 2011;204:566-73.

8. Chaturvedi AK, Anderson WF, Lortet-Tieulent J, et al. Worldwide trends in incidence rates for oral cavity and oropharyngeal cancers. $J$ Clin Oncol 2013;31:4550-9.

9. Ryerson AB, Peters ES, Coughlin SS, et al. Burden of potentially human papillomavirus-associated cancers of the oropharynx and oral cavity in the US, 1998-2003. Cancer 2008;113(10 Suppl):2901-9.

10. Kreimer AR, Clifford GM, Boyle $\mathrm{P}$, et al. Human papillomavirus types in head and neck squamous cell carcinomas worldwide: a systematic review. Cancer Epidemiol Biomarkers Prev 2005;14:467-75.

11. Bruni L, Diaz M, Castellsagué $X$, et al. Cervical human papillomavirus prevalence in 5 continents: meta-analysis of 1 million women with normal cytological findings. J Infect Dis 2010;202:1789-99.
12. Forman D, de Martel C, Lacey CJ, et al. Global burden of human papillomavirus and related diseases. Vaccine 2012;30(Suppl 5): F12-23.

13. Gillison ML, Broutian T, Pickard RK, et al. Prevalence of oral HPV infection in the United States, 2009-2010. JAMA 2012;307:693-703.

14. Shamseer L, Moher D, Clarke M et al. Preferred Reporting Items for Systematic Review and Meta-Analysis Protocols (PRISMA-P) 2015: elaboration and explanation. BMJ 2015;349:g7647.

15. NIH NloH. Quality Assessment Tool for Observational Cohort and Cross-Sectional Studies. 2014. https://www.nhlbi.nih.gov/health-pro/ guidelines/in-develop/cardiovascular-risk-reduction/tools (accessed 1 Oct 2015).

16. Iorio A, Spencer FA, Falavigna M, et al. Use of GRADE for assessment of evidence about prognosis: rating confidence in estimates of event rates in broad categories of patients. BMJ 2015;350:h870.

17. Schwarzer G. General Package for Meta-Analysis. 2015. https:// cran.r-project.org/web/packages/meta/meta.pdf (accessed 12 Feb 2016).

18. Viechtbauer W. Meta-Analysis Package for R. 2015. https://cran r-project.org/web/packages/metafor/metafor.pdf (accessed 12 Feb 2016).

19. Matos LL, Miranda GA, Cernea CR. Prevalence of oral and oropharyngeal human papillomavirus infection in Brazilian population studies: a systematic review. Braz J Otorhinolaryngol 2015;81:554-67. 